\title{
Genetic Characterization of Feline Calicivirus From China Reveals That Amino Acid Mutations at 441- 443 in The ORF2 E Region Are Related to Pathogenicity
}

\section{Xuejiao Liu ( $1055336823 @ q q . c o m)$}

Qingdao Agricultural University https://orcid.org/0000-0002-1964-9585

\section{Weijie Tao}

Qingdao Agricultural University

\section{Yongle Yu}

Qingdao Agricultural University

\section{Haiyan Yang}

Qingdao Agricultural University

Hu Shan

Qingdao Agricultural University

Chuanmei Zhang

Qingdao Agricultural University https://orcid.org/0000-0001-9055-475X

\section{Research Article}

Keywords: Feline calicivirus, Isolation and identification, Genetic analysis, Animal regression experiment

Posted Date: November 16th, 2021

DOI: https://doi.org/10.21203/rs.3.rs-1049525/v1

License: (c) (i) This work is licensed under a Creative Commons Attribution 4.0 International License.

Read Full License 


\section{Abstract}

We studied the diversity and regularity of FCV variations in two new feline calicivirus (FCV) isolates QD-7 and QD-164. We compared the genomes of these new strains with 39 from the NCBI database including isolates from China, United States, Germany, South Korea, the United Kingdom and Japan. The nucleotide sequence identities ranged from $75-88 \%$ indicating a high degree of variability. These variations were not related to distributions of the virus by time of isolation and geographical location. Cats that were experimentally infected with the new isolate QD-164 showed typical clinical symptoms of sneezing, fever and conjunctivitis and all recovered within 30 days. In contrast, QD-7 infections were asymptomatic and the virus was cleared within 16 days. These results indicate that QD-7 and QD-164 were naturally attenuated strains. NNS mutations that are characteristic of highly virulent strains at positions 441-443 were absent from QD-7 while QD-164 possessed an $\mathrm{N}$ at position 442. This indicated that mutations in regions 441-443 may be linked to disease severity.

\section{Introduction}

Feline calicivirus (FCV) is a highly contagious virus that causes mild to severe respiratory infections and oral disease in cats. It is classified in the genus Vesivirus of the Caliciviridae and structurally is a symmetrical icosahedron of 35-39 $\mathrm{nm}$ in diameter and non-encapsulated [1]. The $5.3 \mathrm{~kb}$ FCV RNA genome contains three open reading frames (ORF 1 - 3) that are positioned at the 5 'end of the genome and account for $2 / 3$ of its total size [2]. ORF1 encodes non-structural proteins including proteases that cleave the encoded polyproteins and RNA-dependent RNA polymerases [3]. The capsid proteins are processed by the protease encoded at the 3 ' terminus of ORF1 and this step is essential for viral assembly. Capsid proteins VP1 and VP2 are encoded in ORFs 2 and 3, respectively [4]. Capsid proteins are directly linked to viral host range and infectivity and 6 specific regions of VP1 (A - F) have been functionally defined. In particular, Region $\mathrm{E}$ is a hypervariable segment containing major $\mathrm{B}$ cell epitopes and is used as a reference for strain typing [3].

Cats are the primary hosts of FCV and the virus causes clinical symptoms of oral ulcers, stomatitis, gingivitis and increased oral and nasal secretions [5] as well as upper respiratory tract disease and claudication [6, 7]. These infections occur with high incidence but low mortality [8, 9]. However, there are currently numerous highly virulent FCV strains $[10,11,12]$ that can cause a fatal systemic disease (VSD) $[13,14,15]$.

FCV replication is error-prone due to the lack of proof-reading ability by RNA polymerases and this leads to high sequence variability and antigenic variation [16]. The mutability of FCV strains explains the persistence of FCV in carriers and the emergence of strains associated with severe VSD. Protection against infection can be provided by vaccination but the vaccine is composed of a limited number of strains (F9 and 255) but these do not provide complete protection $[17,18]$. A pan-FCV vaccine is therefore needed and the genomic regions that possess the antigenic variation must be identified. 
In this study, we isolated two FCV strains from cat nasal swabs using F81 cell culture and performed full gene amplification and sequencing. We compared these strains with 39 FCV reference strains from China, the United States, Germany, South Korea, the United Kingdom and Japan that included the vaccine strains F9 and the domestic vaccine strain 255 [19]. Finally, animal regression tests were carried out, and the results showed that the virulence of strain QD-164 was significantly higher than that of strain QD-7. This study will provide materials for further study on FCV mechanisms of variation to search for key mutation sites related to FCV virulence.

\section{Material And Methods}

\subsection{Animals}

Our experiments included six newborn female farm cats that were randomly divided into two groups at 40 days of age and were allowed free access to feed and water and were separately housed.

\subsection{Sample collection}

Clinical samples were selected from cats at Pet Hospitals in Qingdao from 2019 to 2020 that were suspected of FCV infection and displayed symptoms of oral ulcer, conjunctivitis, claudication and pneumonia. Samples were obtained using sterilized cotton swabs from the mucosa of the mouth corner and nose alar and quickly put into sterilized PBS and stored at $-80{ }^{\circ} \mathrm{C}$.

\subsection{Virus isolation}

In vitro virus isolation utilized F81 cells cultured in Dulbecco's modified Eagle medium (Bl, Jiangsu, China) supplemented with $10 \%$ foetal bovine serum (AusGeneX, Molendinar, Australia). Cells were grown at 37 ${ }^{\circ} \mathrm{C}$ in a $5 \% \mathrm{CO}_{2}$ atmosphere and these were the standard conditions used for cultivation. Swab sample liquid was centrifuged at $3000 \mathrm{rpm}$ for $15 \mathrm{~min}$ and $500 \mu \mathrm{L}$ of RT-PCR-positive samples (see below) were passed through $0.22 \mu \mathrm{m}$ filters and then used to inoculate $90 \%$ confluent F81 cells in 6-well plates and incubated as per above (standard conditions). After an adsorption period of $60 \mathrm{~min}$, DMEM supplemented with $1 \%$ FBS was added. Cell cultures were monitored every day for cytopathic effects (CPE). Cultures lacking CPE after $3 \mathrm{~d}$ were centrifuged and supernatants were used to infect new monolayers. The sample was considered negative if no CPE was observed after 3 passages. Cells displaying CPE were freeze-thawed 3 times to release virus [19]. The supernatants from CPE-positive cultures were negatively stained with $0.5 \%$ phosphotungstic acid and observed by electron microscopy using standard procedures [19]. FCV samples were plaque-purified by infecting F81 monolayers in 6-well plates for $1 \mathrm{~h}$ and then adding DMEM solid medium. After three plaque purifications, the virus stored at $-80^{\circ} \mathrm{C}$ until use.

\subsection{RNA extraction and nested RT-PCR}

Total RNA was extracted by using a MiniBEST Viral RNA/DNA Extraction Kit 5.0 (Takara Biotechnology, Qingdao, China) according to the manufacturer's instructions. The samples were initially screened for the 
presence of FCV using nested RT-PCR with the external primer pair Cali1 and Cali2 and internal primer pair Cali3 and Cali4 that targeted a 467 bp portion of the ORF2 gene [6]. DNAStar software was used to splice sequences (Madison, WI, USA).

\subsection{Indirect immunofluorescence (IFA)}

F81 cells were grown as per above in 6-well plates and IFA tests were performed when CPE appeared and the cells had not yet detached. In brief, the cells were washed $3 \times$ with PBS and fixed with $500 \mu \mathrm{L}$ methanol: acetone $1: 3$ at $4{ }^{\circ} \mathrm{C}$ for 30 min and then washed $2 \times$ with PBS. $500 \mu \mathrm{L} 1 \%$ bovine serum albumin (BSA) was added and the plates were incubated as per above $30 \mathrm{~min}$ and then washed $2 \times$ with PBS. Primary antibody (anti-FCV antibody ab33990 [FCV1-43], Abcam, Cambridge, UK) at 1:100 dilution in $500 \mu \mathrm{L}$ was added and the plates were incubated as per above for $1 \mathrm{~h}$ and then washed $3 \times$ with PBS. Secondary antibody (Goat anti-mouse IgG $(H+L)$ FITC, Affinity Biosciences, Jiangsu, China) at 1:100 was added in $500 \mu \mathrm{L}$ to each well and incubated as per above for $1 \mathrm{~h}$ and then washed $3 \times$ with PBS. The cells were viewed under a fluorescent microscope. Uninfected cells served as negative controls.

\subsection{Virus titer determination}

Viral titers were measured using F81 cells in 96 -well plates at $1.5 \times 10^{5}$ cells $/ \mathrm{mL}$ using $100 \mu \mathrm{L}$ per well and cultured as per above for $48 \mathrm{~h}$. Dilutions of FCV were added and allowed to infect for 60 min and the supernatants were then removed and replaced with $200 \mu \mathrm{L}$ DMEM containing FBS. The cells were observed every $12 \mathrm{~h}$ for $5-7 \mathrm{~d}$ and the $50 \%$ tissue culture infective dose $\left(\mathrm{TCID}_{50}\right)$ was calculated using the Reed-Muench method.

\subsection{Sequencing and phylogenetic analyses}

PCR primers F/R1, F/R2 and F/R3 were designed according to conserved fragments of full-length FCV sequences (Table 1) and were designed using Primer 5.0 software. Primers P1P2-F/R, P3P4-F/R and P5P6-F/R have been previously published [19]. In this study, the PCR products were named P1, P2 and P3. Primers were synthesized by Tsingke Biotech (Qingdao, China) (Table 1). PCR reactions were performed in a $25 \mu \mathrm{L}$ reaction mixture containing $2 \mu \mathrm{L}$ template RNA, $12.5 \mu \mathrm{L}$ of $2 \times$ One Step Mix (Dye Plus) and $1.25 \mu \mathrm{L}$ One Step Enzyme Mix (Vazyme Biotech, Nanjing, China), $1 \mu \mathrm{L}$ each primer (10 $\mu \mathrm{M}), 7.25 \mu \mathrm{L}$ RNase-free $\mathrm{ddH}_{2} \mathrm{O}$. The cycling conditions were as follows: $50{ }^{\circ} \mathrm{C} 30 \mathrm{~min}, 94{ }^{\circ} \mathrm{C} 3 \mathrm{~min}, 30$ cycles of $94{ }^{\circ} \mathrm{C}$ $30 \mathrm{~s}, 59{ }^{\circ} \mathrm{C} 30 \mathrm{~s}$ and $72{ }^{\circ} \mathrm{C} 4 \mathrm{~min}$ and a final step at $72{ }^{\circ} \mathrm{C}$ for $10 \mathrm{~min}$. The corresponding reference strains that had been cataloged from 1958 to 2019 were downloaded from the NCBI GenBank (https://www.ncbi.nlm.nih.gov/genbank/) (Table 2). These strains included 17 from China, 16 from the United States, 2 from Germany, 2 from South Korea and 1 was each from the United Kingdom and Japan. The FCV-F9 strain isolated in the United Kingdom in 1958 was a primary vaccine strain abroad and the 2014 U.S. strain FCV-255 was used as a domestic vaccine strain. 
Table 1

Primers used in this study

\begin{tabular}{|lll|}
\hline Primer name & Sequence $\left(\mathbf{5}^{\prime} \mathbf{-} \mathbf{3}^{\prime}\right)$ & Location $(\mathbf{n t})$ \\
\hline P1P2-F & AAAGAAATTTGAGACAATGT & $40-2421$ \\
\hline P1P2-R & ACACCGAATTAACGGTTACCACAT & \\
\hline P3P4-F & GTGGTAACCGTTAATTCGGTGTTT & 2473-5273 \\
\hline P3P4-R & CACGTTAGCGCAGGTTGAGCAC & \\
\hline P5P6-F & TGCTCAACCTGCGCTAACGTGC & $5326-7560$ \\
\hline P5P6-R & CCTGGGGTTAGGCGC & \\
\hline F1 & GAAGGTAACTGTGTCCAAACTGCAT & $2290-2680$ \\
\hline R1 & CAAACTGCGTTACAACATGCTCAT & \\
\hline F2 & GCGGTTGAGTATGAGGAGCTT & $5140-5462$ \\
\hline R2 & TTGGTTACAATCCCACACAGTT & \\
\hline F3 & GAACAACCAAGTTGAGCAATTTAAC & $7436-7680$ \\
\hline R3 & GTTAGGCGCAGGTGCGGCA & \\
\hline Cali1 & AACCTGCGCTAACGTGCTTA & 924 \\
\hline Cali2 & CAGTGACAATACACCCAGAA & \\
\hline Cali3 & TGGTGATGATGAATGGGCATC & 477 \\
\hline Cali4 & ACACCAGAGCCAGAGATAGA & \\
\hline
\end{tabular}


Table 2

Reference strains

\begin{tabular}{|c|c|c|c|c|}
\hline Number & Strain & Accession number & Location & Year \\
\hline 1 & Urbana2003 & L40021 & U.S. & 2003 \\
\hline 2 & USDA & AY560118 & U.S. & 2006 \\
\hline 3 & UTCVM-H1 & AY560116 & U.S. & 2005 \\
\hline 4 & UTCVM-H2 & AY560117 & U.S. & 2005 \\
\hline 5 & UTCVM-NH1 & AY560113 & U.S. & 2005 \\
\hline 6 & UTCVM-NH2 & AY560114 & U.S. & 2005 \\
\hline 7 & UTCVM-NH3 & AY560115 & U.S. & 2005 \\
\hline 8 & 2280 & KC835209 & U.S. & 2013 \\
\hline 9 & 3786 & JX519209 & U.S. & 2014 \\
\hline 10 & 21223 & JX519212 & U.S. & 2014 \\
\hline 11 & FCV-255 & KM111171 & U.S. & 2014 \\
\hline 12 & 5789 & JX519210 & U.S. & 2014 \\
\hline 13 & 1874 & JX519214 & U.S. & 2014 \\
\hline 14 & 20879 & JX519211 & U.S. & 2014 \\
\hline 15 & CFI-68 & U13992 & U.S. & 1998 \\
\hline 16 & CH-JL1 & KJ495728 & Jilin China & 2017 \\
\hline 17 & CH-JL2 & KJ495729 & Jilin China & 2013 \\
\hline 18 & CH-JL3 & KJ495730 & Jilin China & 2013 \\
\hline 19 & CH-JL4 & KT206207 & Jilin China & 2015 \\
\hline 20 & HRB-SS & KM016908 & Heilong jiang China & 2014 \\
\hline 21 & WZ-1 & KX371573 & Heilong jiang China & 2016 \\
\hline 22 & TFHLJ-8 & KJ944377 & Heilong jiang China & 2014 \\
\hline 23 & TIG-1 & KU373057 & Heilong jiang China & 2016 \\
\hline 24 & $\mathrm{XH}$ & KX371572 & Heilong jiang China & 2016 \\
\hline 25 & SH2014 & KT000003 & Shanghai China & 2014 \\
\hline 26 & $\mathrm{SH}$ & KP987265 & Shanghai China & 2015 \\
\hline 27 & $\mathrm{CH}-\mathrm{GD}$ & GU214989 & Guangdong China & 2009 \\
\hline
\end{tabular}




\begin{tabular}{|lllll|}
\hline Number & Strain & Accession number & Location & Year \\
\hline 28 & YH16 & KX815169 & Beijing China & 2015 \\
29 & GX01-13 & KT970059 & Guangxi China & 2013 \\
30 & GX2019 & MK867378 & Guangxi China & 2019 \\
31 & FB-NJ-13 & KM111557 & Jiangsui China & 2014 \\
32 & HB-S4 & KT267162 & Hubei China & 2015 \\
33 & 12Q087-1 & KJ572400 & South Korea & 2012 \\
34 & 12Q087-5 & KJ572401 & South Korea & 2014 \\
35 & FCLF4 & D31836 & Japan & 2014 \\
36 & F9 & M86379 & U.S. & 1958 \\
37 & F65 & AF109465 & United Kingdom & 1990 \\
38 & FCV 2024 & AF479590 & Germany & 2002 \\
39 & FCV-DD-2006-GE & DQ424892 & Germany & 2006 \\
\hline 2.8 Analysis Of amino aCid mutation Sites &
\end{tabular}

The FCV ORF2 encodes the major capsid protein and the E region (amino acids 426-480) is highly variable. This region for $22 \mathrm{FCV}$ strains was selected and compared with the FCV strains we isolated for this study and with vaccine strain F9 as a reference. The strains UTCVM-H1, UTCVM-H2 and 2280 were VSD strains.

\subsection{Animal regression experiments}

Oral, nasal and rectal swabs and plasma samples of experimentally-infected cats were collected before the start of the study and screened for the presence of FCV, feline leukemia virus (FeLV), feline parvovirus (FPV), feline herpes virus type 1 (FHV-1) and feline coronavirus (FCoV) using PCR as previously reported $[20,21,22]$. Antibody levels for FCV and FHV-1 were also determined from serum samples. The animals used for challenge experiments all tested negative for these tests. Six Chinese garden cats at 40 days of age were randomly divided into challenge group and control group, with three cats in each group. In the challenge group, a total of $2 \mathrm{~mL}$ of QD-164 virus stock ( $\left.\mathrm{TCID}_{50} 10^{-6.5} / 0.1 \mathrm{~mL}\right)$ was administered to the eyes, noses and mouths after mild anesthesia. The control group was inoculated with $2 \mathrm{~mL}$ normal cell culture medium in the same method.

Clinical signs of infection were monitored daily and the food and water intake as well as temperature and weight were monitored. On days 3, 5, 9, 14 and 21 after challenge, oral and nasal swabs and anal swabs were collected to monitor virus shedding and blood samples were collected to monitor antibodies. 


\section{Results}

\subsection{Virus isolation}

In this study, our initial screening of farm cats resulted in the identification of FCV strains QD-164 and QD7 and when inoculated onto F81 monolayers caused the cells to round-up and become wrinkled and formed grape-like clusters. Upon subculture of viral supernatants, typical cytopathic lesions could be observed in the monolayers (Figure 1). The FCV virus particles were spherical and non- enveloped and consistent with the structural characteristics of the Caliciviridae (Figure 2). Infection of F81 cells with these viral strains was also monitored using IFA directed against the viral capsid protein epitope of FCV (Figure 3). Together, these results indicated that our viral isolates QD-164 and QD-7 were FCV members.

\subsection{Virus titer determination}

Viral titers were determined using 96 -well culture plate monolayers. The $\operatorname{TCID}_{50}$ of viral samples after 3 rounds of purification were $10^{-6.5} / 0.1 \mathrm{~mL}$ and $10^{-4.2} / 0.1 \mathrm{~mL}$ for QD-164 and QD-7, respectively.

\subsection{Sequencing and phylogenetic analyses}

We performed complete sequence analysis of our 2 new isolates QD-164 and QD-7 and compared the genomes to the FCV strains in the NCBI database (Figure 4). QD-7 had the highest nucleotide sequence identity (82.0\%) with FB-NJ-13 and the lowest (75.8\%) with UTCVM-H1 while QD-164 was most closely related to $\mathrm{CH}-\mathrm{JL} 4$ (85.5\%) and more distantly related (76.4\%) to CH-GD and HB-S4. The 2 new strains were only $77.0 \%$ identical while homology with the vaccine strains F9 and FCV-255 ranged from 76 to $78.8 \%$. Overall, the nucleotide identities between new strains and the $39 \mathrm{FCV}$ catalogued strains ranged from 75.6 to $97.2 \%$ and most were $<80 \%$ and only 3 strains exceeded $90 \%$ with only 4 were $>80 \%$.

This collection of 41 FCV strains we analyzed could be divided into two large branches where QD-7 occupied the same large branch as two Korean strains and six other Chinese strains. QD-164 was located in the large clade that encompassed many countries but in the same small clade as the Chinese isolates GX2019 and CH-JL4 and the German strain FCV-DD-2006-GE and the US strain UTCVM-H2. This suggested relatively close evolutionary relationships for the latter 5 strains. The vaccine strain $\mathrm{F} 9$ was the most closely related to the American strain UTCVM-NH2 while vaccine strain FCV-255 was highly homologous to the American strains FCV-2024 and USDA and these showed 99.2 -99.9\% nucleotide identities indicating a likely common ancestor (Figure 5).

\subsection{Analysis of amino acid mutation sites}

We screened our isolates for the presence of mutations in the highly variable region E of ORF2 (amino acids 426-480). A comparison with the other database strains indicated that common mutations were aa E464K and G465S compared with reference strain F9(Figure 6). In addition, the highly virulent (VSD) strains UTCVM-H1 and UTCVM-H2 as well as TFHLJ-8 and 2280 possessed an NNS alteration at 441-443 compared with TGN for the reference strain as previously reported [23]. This set of mutations were not present in the new strains QD-7 and QD-164 and indicated that these strains were most likely not VSD. 
The sequence of TFHLJ-8 was directly submitted to the database NCBI without description of related symptoms so it could not be linked to VSD. A direct causality between these E region mutations and VSD strains has not yet been found.

\subsection{Animal regression experiments}

We assessed the ability of our new strains QD-7 and QD-164 to cause disease by direct infection with test animals. The weight gains for the viral challenge group lagged significantly behind the control animals and overall, the challenged cats lost weight compared with controls. The animals in the challenge group also presented with fever within $7 \mathrm{~d}$ of challenge while the controls maintained the normal body temperature. The challenge group also generated a maximal antibody response to infection at day 21 when monitored at days $3,5,9,14$ and 21. PCR detection of FCV in the test animals indicated that the virus was present for $16 \mathrm{~d}$ from oral and nasal swabs and FCV was detected in a rectal swab of only one test animal. The controls were negative for the presence of the virus (Figure 7).

The QD-164 challenge group had fever within $7 d$ following challenge and some had diarrhea. Sneezing commenced from day 8 and this was accompanied by nasal discharges. On day 9 , conjunctivitis began to appear, with red eyelids and depression. On the 12th day, symptoms worsened and purulent secretions were present. All the animals in the challenge group showed typical clinical symptoms and eventually recovered within $20 \mathrm{~d}$ without treatment but nasal discharge and sneezing lasted for 30 days. PCR analysis of nasal swabs indicated that the virus was cleared by day 16 . There were no clinical symptoms for animal infection with QD-7 although the pattern of viral shedding was similar to that of QD-164 (Figure 8).

\section{Discussion}

FCV is a serious pathogen of cats and poses a great threat to these animals. The presence of a FCV universal vaccination program with the current vaccine strains would still result in cats exhibiting clinical symptoms of infection [24]. In addition, the presence of highly virulent VSD strains often result in high levels of mortality and this cannot be prevented by the current vaccines. Our analysis of homology and genetic relatedness between $41 \mathrm{FCV}$ from China and abroad indicated that even FCV isolates from the same year and country were not highly homologous and were distributed along different branches of the evolutionary tree. This suggested a wide diversity and high level of uncertainty to predict FCV strain variation for the purpose of vaccine development. However, the German strain FCV 2024 (2002) and the US strain USDA (2006) were $99.9 \%$ identical indicating that the variations are not significantly related in time and space. Similar examples include the 2016 Chinese isolate TIG-1 and 2003 U.S. isolate Urbana 2003 (97.2 \% identity) and the 1992 British isolate F9 and 2005 U.S. isolate UTCVM-NH2 (98.2 \% identity).

FCV strains from China were distributed across two large clades without obvious clustering. The 2 FCV strains isolated in this study had obvious clustering with other Chinese strains in the evolutionary tree, and had higher homology with FCV strains isolated in Northeast China. Overall, with the exception of TIG1 and TFHLJ-8 from China, the Asian FCV strains clustered in the lower part of the tree while most of the 
American strains clustered in the upper part. This suggested that there may be significant evolutionary differences between continents but that FCV strains from the same continent still have similar evolutionary outcomes.

We did not find any evidence for temporal or spatial association between FCV from the same area and this was consistent with previous reports $[16,19,25]$. The 17 Chinese isolates were distributed across different provinces and the homology for each strain was not significantly related to its location and time of isolation. Strains SH and SH2014 from Shanghai were a typical example and these displayed only $76.3 \%$ nucleotide identity while the Guangdong isolates from 2009 had relatively high homology with SH (84.3 \%). The 17 Chinese FCV strains except for TFHLJ-8 and TIG-1 had relatively high homology while all the other strains had levels ranging between 75 and $85 \%$. The latter were close to the range of homology for the whole group of 41 strains (75 to $88 \%$ ). This indicated that the degree of FCV variation in China was about the same as the global level and did not deviate from the general trend.

The two vaccine strains $(F 9,255)$ were most closely related to USDA and FCV 2024 that possessed levels $>90 \%$ identity with FCV-255. Only strain UTCVM-NH2 was > $90 \%$ related with F9. In general, homology between the vaccine and other strains was generally low. Therefore, none of the vaccine strains used at home and abroad can provide good protection and new vaccine strains must be developed.

In this study, our new isolate QD-164 was the only one to produce the typical symptoms of FCV infection. These included runny nose, fever, sneezing and conjunctivitis, but these symptoms all healed spontaneously after a period of time. The virus was also cleared in $16 \mathrm{~d}$ and this was close to the previously reported minimum detoxification time of $2 \mathrm{wk}$. Strain QD-164 was only transiently present in the test animals. Additionally, FCV antibodies in the test animals decreased to the pre-challenge level within $30 \mathrm{~d}$. Therefore, QD-164 is a natural weak strain isolated from the Qingdao area that causes only mild symptoms whereas QD-7 infections were asymptomatic. The body temperature, body weight and antibody levels in the challenge group were the same as those in the control group. QD-7 was significantly different from QD-164 in the evolutionary tree, which was located in a separate branch. QD164 had N-442 mutation, and QD-7 had no difference from the reference strain. Therefore, the mutation at 441-443 may be the key to predict whether the strain has capability of VSD. We speculated that the closer the amino acid mutation at position 441-443 was to NNS, the stronger the virulence of the strain would be. When the NNS mutation completely appeared, the VSD strain might be generated. However, this hypothesis still needs to be confirmed by extensive sequencing and related animal tests.

As an RNA virus, FCV has evolved rapidly and is extremely diverse as can be seen in the phylogenetic analysis of genomic sequence data. This strain diversity is a major challenge for vaccine cross-protection [11]. Vaccines against FCV are available as attenuated and as combined with FPV and FHV vaccines. Vaccines are required to be able to protect against all wild viruses. However, due to the diversity of FCV strains, there is still no FCV vaccine that can play a complete protective role [26]. Interestingly, the overexpression of the apoptosis inhibitor survivin has a negative effect on FCV replication [27]. FCV is 
still changing and we should strengthen the research on the mechanism of FCV mutation in order to better prevent and control FCV infection in the future.

\section{Declarations}

\section{Ethics approval and consent to participate}

This study was conducted according to the animal welfare guidelines of the World Organization for Animal Health (Terrestrial Animal Health Code) and approved by the Committee on ethics from the College of Veterinary Medicine, Qingdao Agricultural University (Qingdao, China).

\section{Consent for publication}

Not applicable.

\section{Availability of data and materials}

Not applicable.

\section{Competing interests}

The authors declare that they have no competing interests.

\section{Funding}

The 2020 professional degree graduate teaching case building project of Qingdao Agricultural University (QNYAL2002).

\section{Authors' contributions}

Acquisition, analysis, interpretation of data, Liu X.J., Tao W.J.; Writing-Original Draft Preparation, Liu X.J.; Revision \& Editing, Zhang C.M., Yu Y.L., Yang H.Y.; Supervision, Shan H.

\section{Acknowledgements}

The authors thank all participating veterinarians for collecting the samples and case information, ZiHe Li, YanLei Cui, JiaHui Liu for excellent laboratory assistance. This research was supported by the 2020 professional degree graduate teaching case building project of Qingdao Agricultural University (QNYAL2002).

\section{References}

1. Zang M, He W, Du F, Wu G, Wu B, Zhou Z (2017) Analysis of the codon usage of the ORF2 gene of feline calicivirus. Infect Genet Evol 54:54-59 
2. Fumian TM, Tuipulotu DE, Netzler NE, Lun JH, Russo AG, Yan GJH, White PA (2018) Potential Therapeutic Agents for Feline Calicivirus Infection. Viruses. 10

3. Radford AD, Coyne KP, Dawson S, Porter CJ, Gaskell RM (2007) Feline calicivirus. Vet Res 38:319335

4. Brunet S, Sigoillot-Claude C, Pialot D, Poulet H (2019) Multiple Correspondence Analysis on Amino Acid Properties within the Variable Region of the Capsid Protein Shows Differences between Classical and Virulent Systemic Feline Calicivirus Strains. Viruses. 11.

5. Cui Z, Li D, Yi S, Guo Y, Dong G, Niu J, Zhao H, Zhang Y, Zhang S, Cao L, Wang K, Zhao Y, Hu G (2019) Equine immunoglobulin $F(a b ') 2$ fragments protect cats against feline calicivirus infection. Int Immunopharmacol 75:105714

6. Marsilio F, Di Martino B, Decaro N, Buonavoglia C (2005) A novel nested PCR for the diagnosis of calicivirus infections in the cat. Vet Microbiol 105:1-7

7. Spiri AM, Meli ML, Riond B, Herbert I, Hosie MJ, Hofmann-Lehmann R (2019) Environmental Contamination and Hygienic Measures After Feline Calicivirus Field Strain Infections of Cats in a Research Facility. Viruses. 11.

8. Berger A, Willi B, Meli ML, Boretti FS, Hartnack S, Dreyfus A, Lutz H, Hofmann-Lehmann R (2015) Feline calicivirus and other respiratory pathogens in cats with Feline calicivirus-related symptoms and in clinically healthy cats in Switzerland. BMC Vet Res 11:282

9. Radford AD, Addie D, Belak S, Boucraut-Baralon C, Egberink H, Frymus T, Gruffydd-Jones T, Hartmann K, Hosie MJ, Lloret A, Lutz H, Marsilio F, Pennisi MG, Thiry E, Truyen U, Horzinek MC (2009) Feline calicivirus infection. ABCD guidelines on prevention and management. J Feline Med Surg 11:556564

10. Caringella F, Elia G, Decaro N, Martella V, Lanave G, Varello K, Catella C, Diakoudi G, Carelli G, Colaianni ML, Bo S, Buonavoglia C (2019) Feline calicivirus infection in cats with virulent systemic disease, Italy. Res Vet Sci 124:46-51

11. Hou J, Sanchez-Vizcaino F, McGahie D, Lesbros C, Almeras T, Howarth D, O'Hara V, Dawson S, Radford AD (2016) European molecular epidemiology and strain diversity of feline calicivirus. Vet Rec 178:114-115

12. Guo H, Miao Q, Zhu J, Yang Z, Liu G (2018) Isolation and molecular characterization of a virulent systemic feline calicivirus isolated in China. Infect Genet Evol 65:425-429

13. Kate FH, Patricia AP, Niels CP, Amy MP, Erin W, Janet EF (2004) An outbreak of virulent systemic feline calicivirus disease. J Am Vet Med Assoc 224:241-249

14. Coyne KP, Jones BRD, Kipar A, Chantrey J, Porter CJ, Barber PJ, Dawson S, Gaskel RM, Radford AD (2006) Lethal outbreak of disease associated with feline calicivirus infection in catsp. Vet Rec 158:544-550

15. Schulz BS, Hartmann K, Unterer S, Eichhorn W, Majzoub M, Homeier-Bachmann T, Truyen U, Ellenberger C, Huebner J (2011) Two outbreaks of virulent systemic feline calicivirus infection in cats in Germany. Berl Munch Tierarztl Wochenschr 124:186-193 
16. Smith SL, Afonso MM, Pinchbeck GL, Gaskell RM, Dawson S, Radford AD (2019) Temporally separated feline calicivirus isolates do not cluster phylogenetically and are similarly neutralised by high-titre vaccine strain FCV-F9 antisera in vitro.J Feline Med Surg,8

17. Pedersen NC, Elliott JB, Glasgow A, Polanda A, Keel K (2000) An isolated epizootic of hemorrhagiclike fever in cats caused by a novel and highly virulent strain of feline calicivirus. Vet Microbiol. 73:281-300

18. Lauritzen A, Jarrett O, Sabara M (1997) Serological analysis of feline calicivirus isolates from the United States and United Kingdom.Vet. Microbiol 56:55-63

19. Zhao Y, Chen X, Ying Y, Wang K, Dong H, Gao C, Yang S, Hu G (2017) Isolation and phylogenetic analysis of three feline calicivirus strains from domestic cats in Jilin Province, China. Arch Virol 162:2579-2589

20. Abd-Eldaim M, Potgieter L, Kennedy M (2005) Genetic analysis of feline caliciviruses associated with a hemorrhagic-like disease. J Vet Diagn Invest 17:420-429

21. Radford AD, Gaskell RM (2011) Dealing with a potential case of FCV-associated virulent systemic disease.Vet Record. 168:585-586

22. Barrera-Vazquez OS, Cancio-Lonches C, Miguel-Rodriguez CE, Valdes Perez MM, Gutierrez-Escolano AL (2019) Survivin Overexpression Has a Negative Effect on Feline Calicivirus Infection.Viruses, 11

23. Liu C, Liu Y, Qian P, Cao Y, Wang J, Sun C, Huang B, Cui N, Huo N, Wu H, Wang L, Xi X, Tian K (2020) Molecular and serological investigation of cat viral infectious diseases in China from 2016 to 2019. Transbound Emerg Dis 67:2329-2335

24. Rong S, Lowery D, Floyd-Hawkins K, King V (2014) Characterization of an avirulent FCV strain with a broad serum cross-neutralization profile and protection against challenge of a highly virulent vs feline calicivirus. Virus Res 188:60-67

25. Pereira JJ, Baumworcel N, Fioretti JM, Domingues CF, Moraes LF, Marinho R, Vieira MCR, Pinto AMV, de Castro TX (2018) Molecular characterization of feline calicivirus variants from multicat household and public animal shelter in Rio de Janeiro, Brazil. Braz J Microbiol 49:777-784

26. Vlasova AN, Halpin R, Wang S, Ghedin E, Spiro DJ, Saif LJ (2011) Molecular characterization of a new species in the genus Alphacoronavirus associated with mink epizootic catarrhal gastroenteritis. J Gen Virol 92:1369-1379

27. Wang K, Du S, Wang Y, Wang S, Luo X, Zhang Y, Liu C, Wang H, Pei Z, Hu G (2019) Wanglsolation and identification of tiger parvovirus in captive siberian tigers and phylogenetic analysis of VP2 gene. Infect Genet Evol 75:103957

\section{Figures}




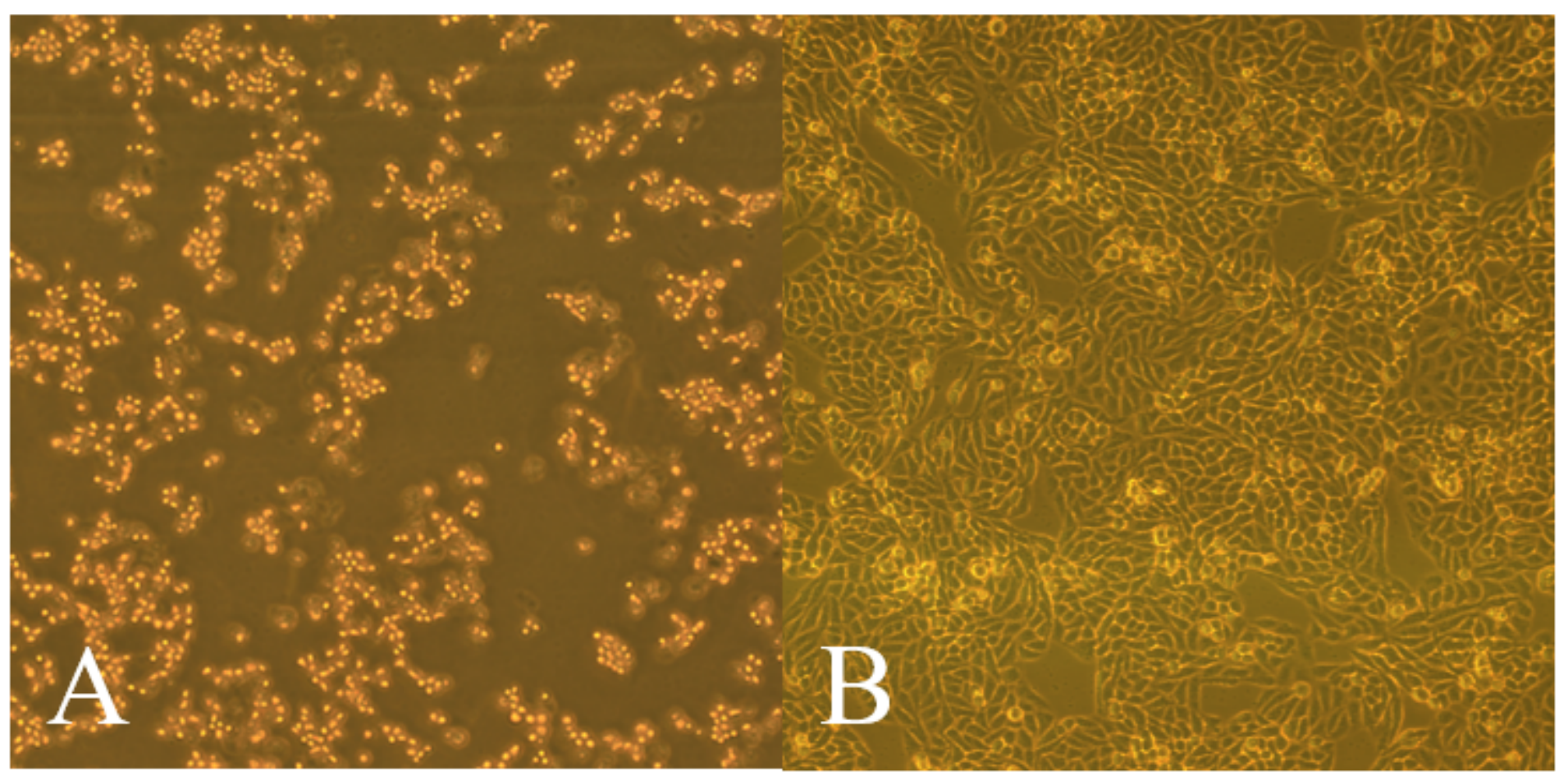

Figure 1

CPE in F81 cell monolayers following infection with strains (A) Infected group and (B) Control group. 


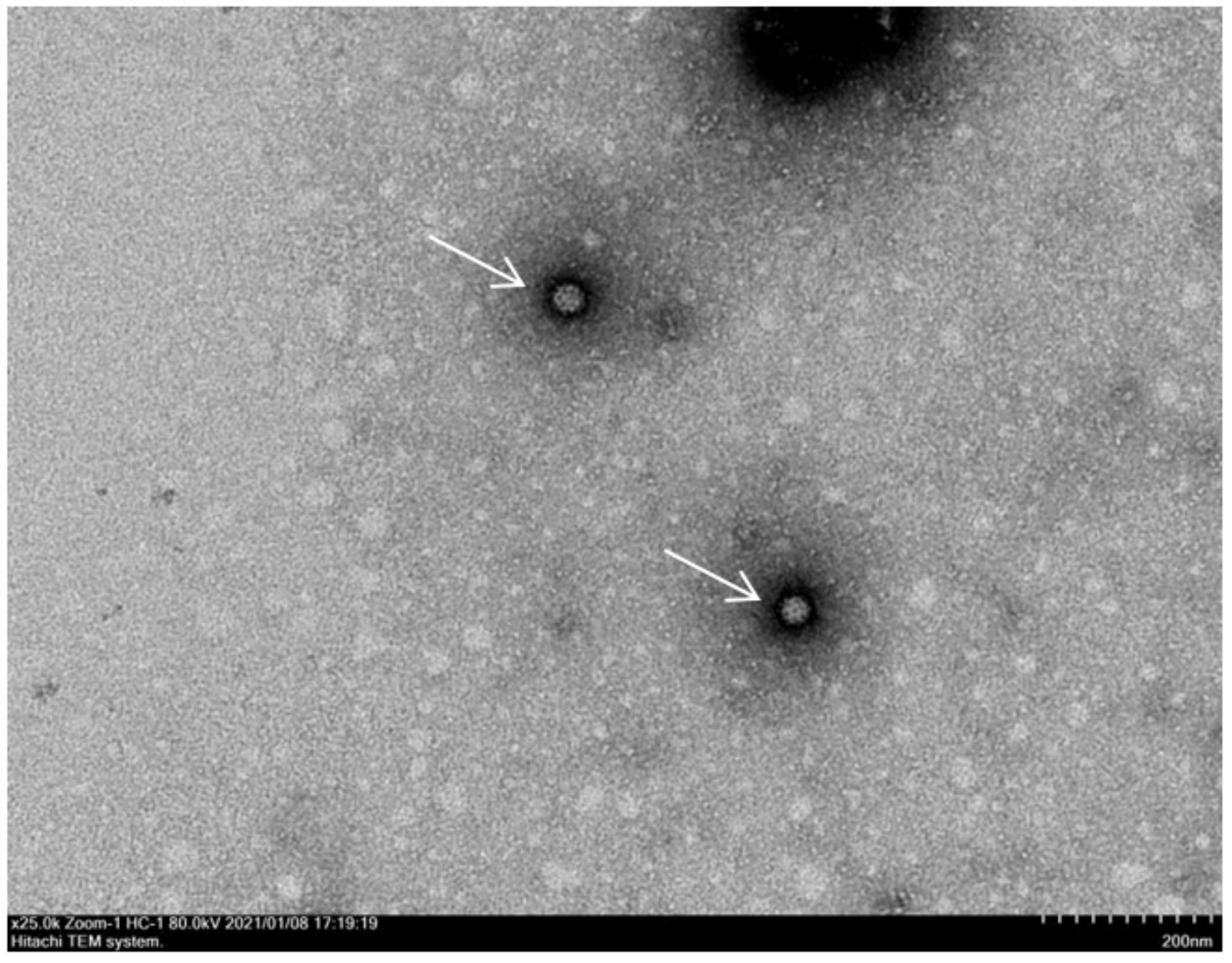

Figure 2

Electron micrographs of strain QD-164 viral particles from infected F81 cells. 


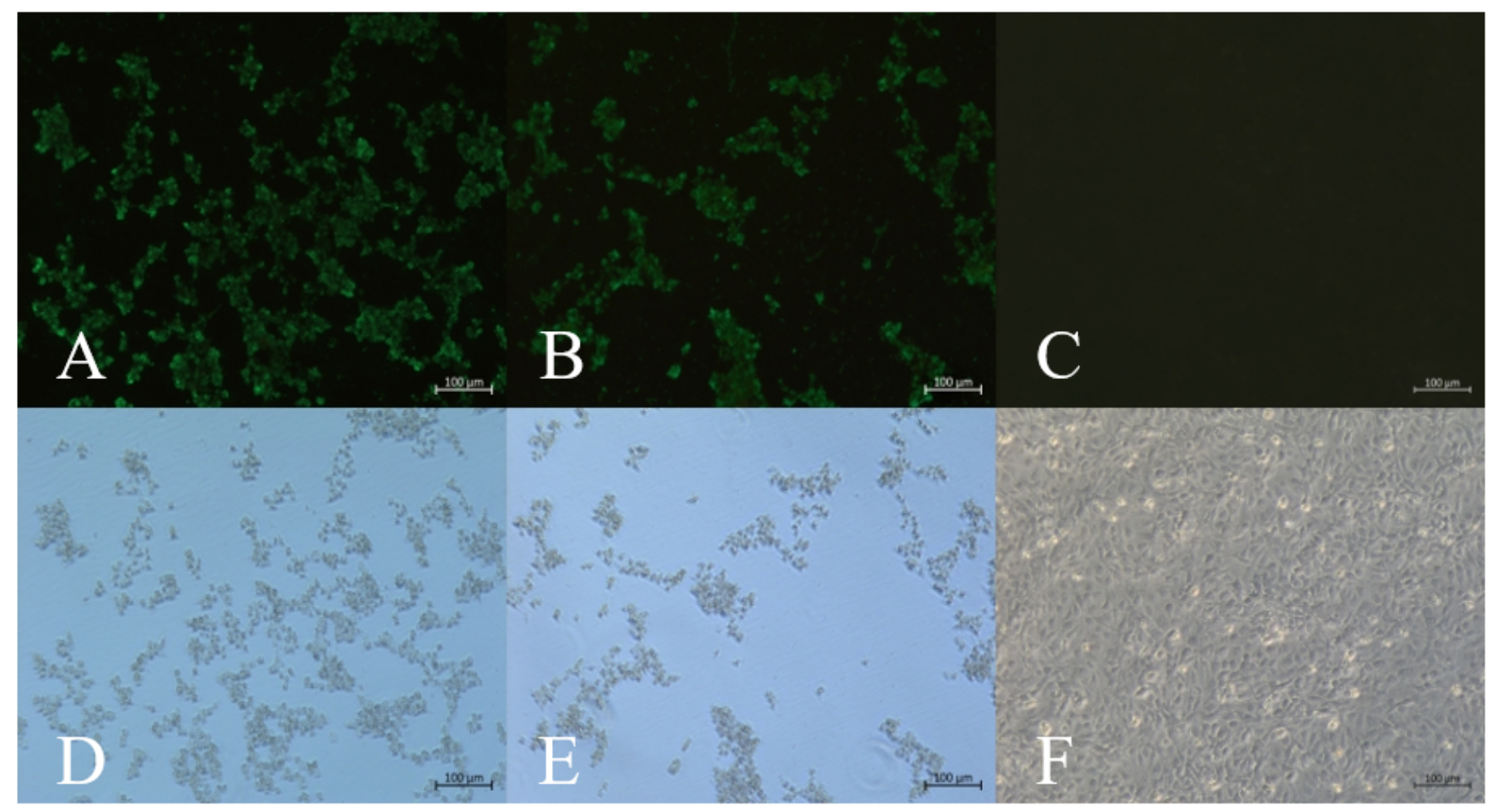

\section{Figure 3}

IFA identification of infected F81 cell monolayers. (A, D) Strain QD-164 (B, E) Strain QD-7 (C, F) Uninfected negative control.

\section{P1 P2 P3}

5000 3000 
Figure 4

PCR amplification of the complete FCV genome for the newly identified strains QD-7 and QD-164. Lane 1, M: DL5000 DNA size marker (Vazyme Biotech); Lane 2, P1; Lane 3, P2; Lane 4, P3.

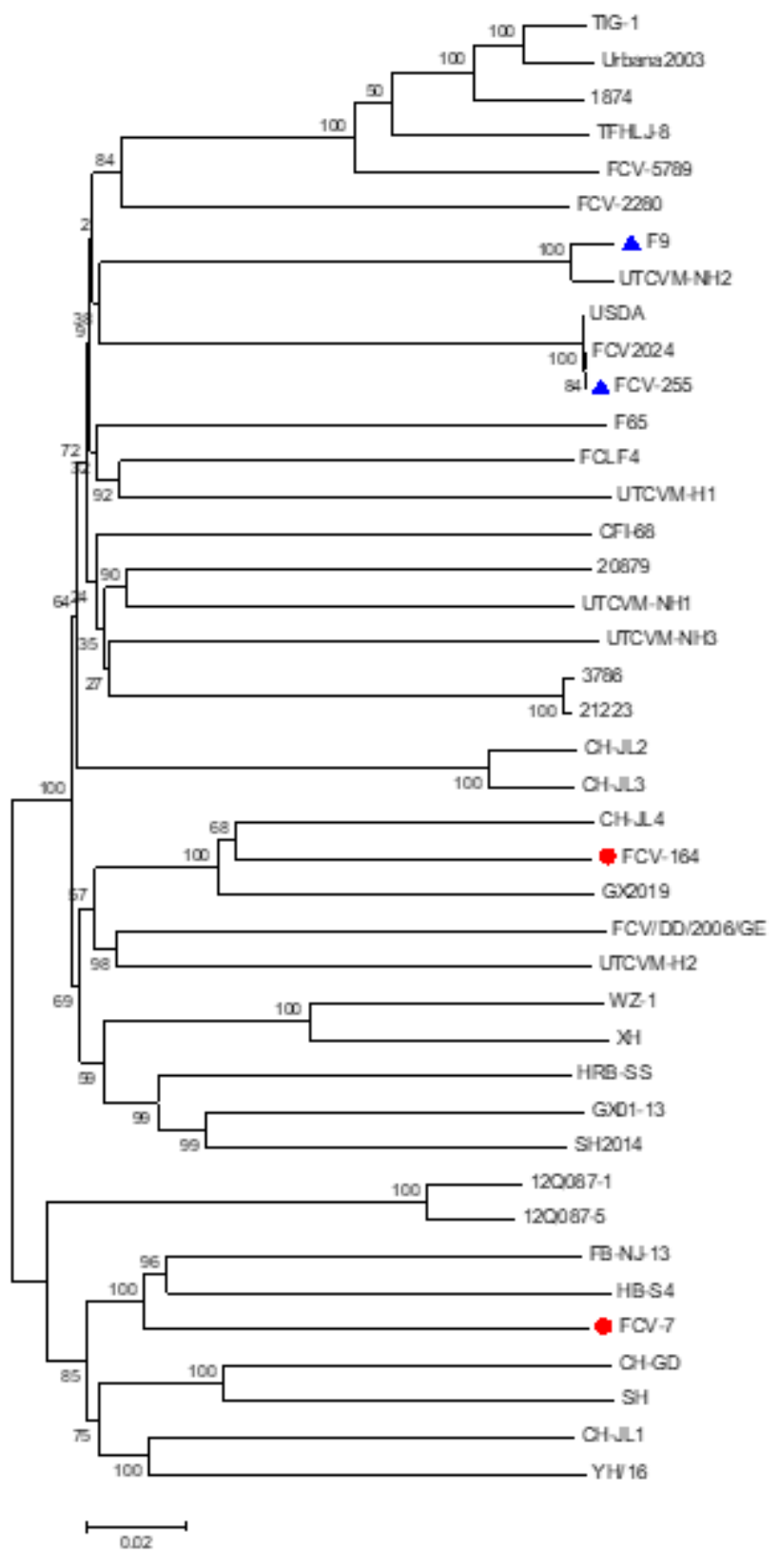

\section{Figure 5}

Genetic and evolutionary analysis of FCV. Strains QD-7 and QD-164 are indicated by the red dots. The blue triangles represent vaccine strains. 


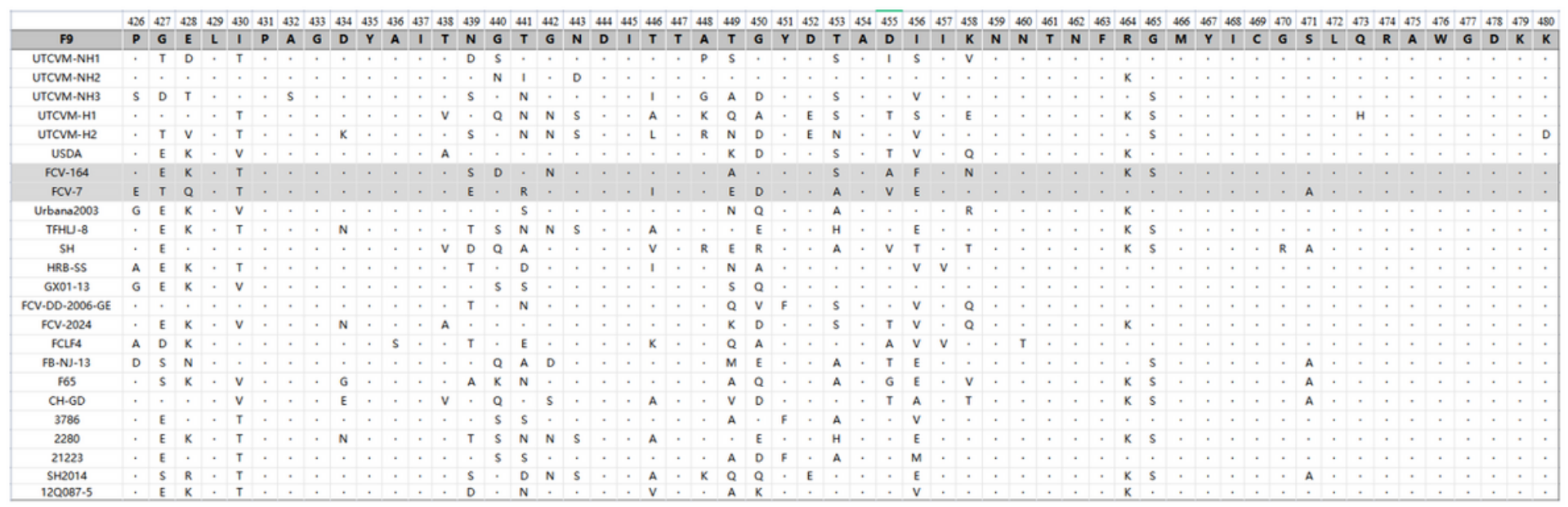

\section{Figure 6}

Analysis of the variation sites in the E region of ORF2 of 25 strains of FCV. Shaded region, new FCV strains QD-7 and QD-164.

A

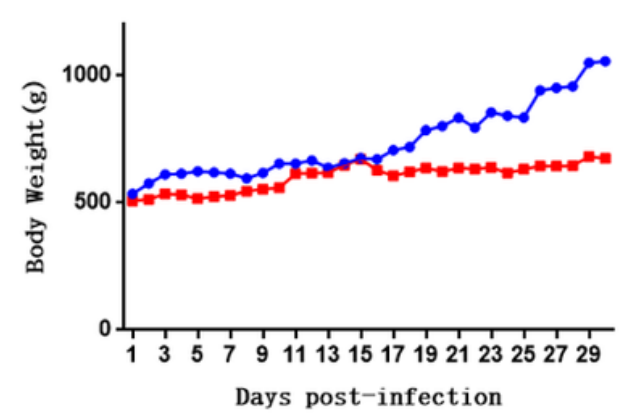

B

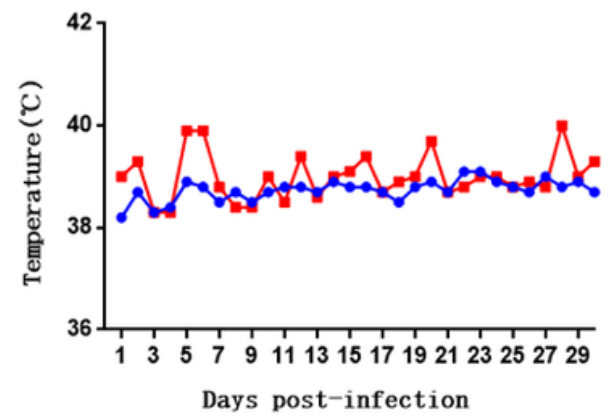

C

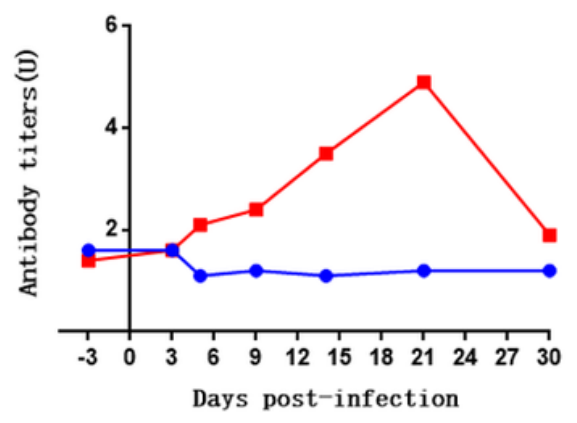

Figure 7

Monitoring of experimentally infected adult cats with the new FCV strains QD-7 and QD-164 for 30 days. Red and blue lines represent infected group and control group, respectively. (A) Body weight (B) Body temperature (C) Presence of serum antibody to FCV IgG. 

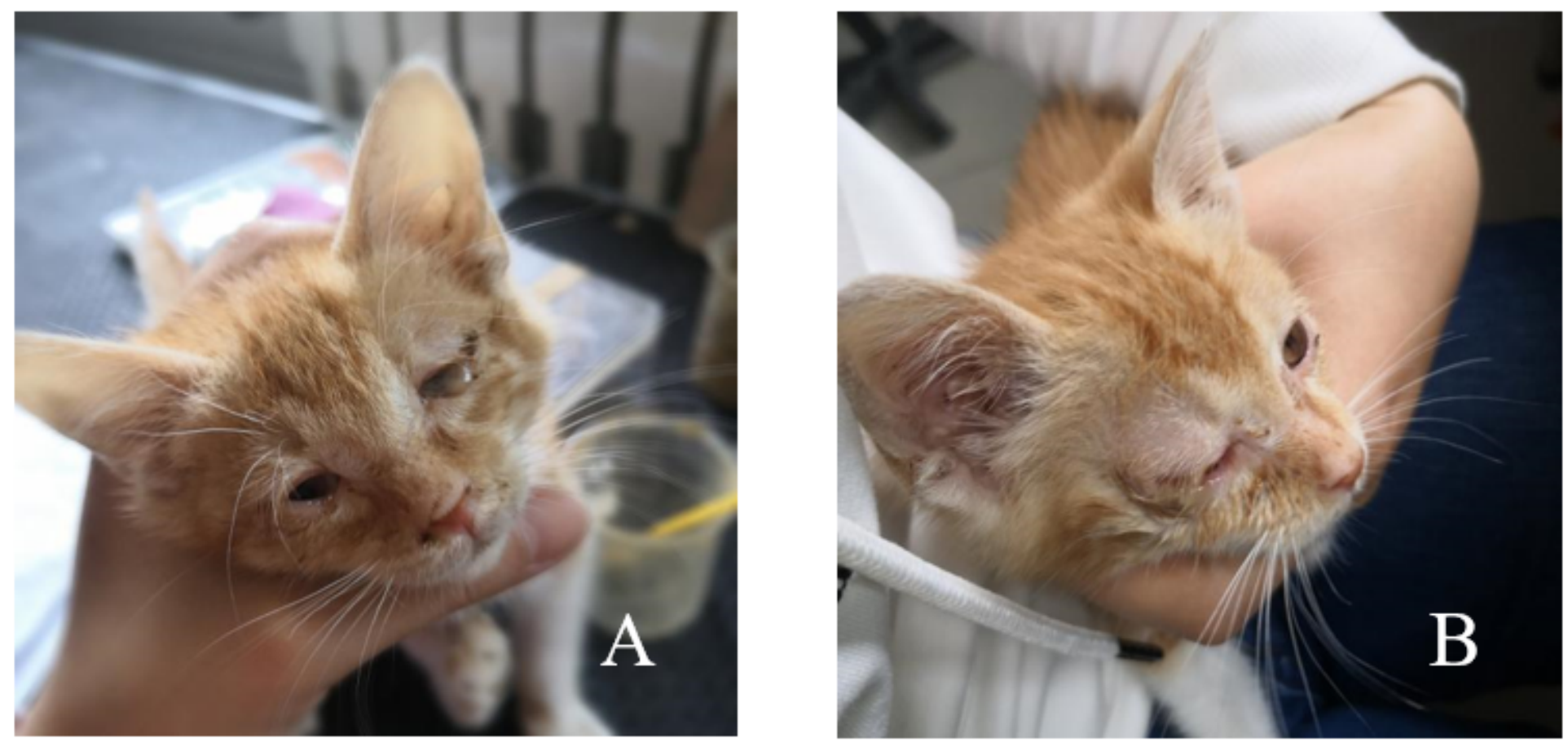

\section{Figure 8}

Clinical symptoms for animals challenged with the virus. (A) Eye secretions and the presence of a yellow purulent discharge (B) Conjunctivitis with swelling, redness and increased secretions in the upper eyelid. 